\title{
Tungsten divertor erosion in all metal devices: lessons from the ITER Like Wall of JET
}

\author{
G.J. van Rooij ${ }^{1 *}$, J.W. Coenen ${ }^{2}$, L. Aho-Mantila ${ }^{3}$, S. Brezinsek ${ }^{2}$, M. Clever ${ }^{2}$, R. Dux ${ }^{4}$, M. \\ Groth $^{5}$, K. Krieger ${ }^{4}$, S. Marsen ${ }^{4}$, G.F. Matthews ${ }^{6}$, A. Meigs ${ }^{6}$, R. Neu ${ }^{4}$, S. Potzel ${ }^{4}$, T. Pütterich ${ }^{4}$, \\ J. Rapp ${ }^{7}$, M.F. Stamp ${ }^{6}$, the ASDEX Upgrade Team ${ }^{4}$, and JET-EFDA Contributors ${ }^{++}$ \\ JET-EFDA, Culham Science Centre, OX14 3DB, Abingdon, UK \\ ${ }^{1}$ Dutch Institute For Fundamental Energy Research, Assoc. EURATOM-FOM, The Netherlands ${ }^{+}$ \\ ${ }^{2}$ Institute of Energy and Climate Research, Forschungszentrum Jülich, Assoc EURATOM-FZJ, Jülich, Germany ${ }^{+}$ \\ ${ }^{3}$ VTT, P.O. Box 1000, FI-02044 VTT, Finland \\ ${ }^{4}$ Max-Planck-Institut für Plasmaphysik, Association EURATOM-IPP, Germany \\ ${ }^{5}$ Aalto University, Association EURATOM-Tekes, Espoo, Finland \\ ${ }^{6}$ Culham Centre for Fusion Energy, EURATOM-CCFE Association, Abingdon, UK \\ ${ }^{7}$ Oak Ridge National Laboratory, Oak Ridge, USA \\ ${ }^{+}$Partner in the Trilateral Euregio Cluster \\ and \\ ${ }^{++}$See App. of F. Romanelli et al., Proceedings of the 23rd IAEA Fusion Energy Conf. 2010, Daejeon, Korea
}

\begin{abstract}
Tungsten erosion in the outer divertor of the JET ITER like wall was quantified by spectroscopy. Effective sputtering yields of typically $10^{-4}$ were measured in L-mode at $\sim 30 \mathrm{eV}$ attached divertor conditions and beryllium was identified as the main cause of sputtering. The signature of prompt redeposition was observed in the analysis of WI $400.9 \mathrm{~nm}$ and WII $364 \mathrm{~nm}$ line ratios and indicative of $>50 \%$ redeposition fractions. Inter- and intra-ELM sputtering were compared for an example of $10 \mathrm{~Hz}$ ELMs with 13 MW NBI heating, in which intra-ELM sputtering was found to dominate by a factor of 5. Nitrogen seeding initially increased the tungsten sputtering threefold due to higher extrinsic impurity levels and effectively reduced the tungsten sputtering when the divertor plasma temperature was decreased from the initial $25 \mathrm{eV}$ down to $15 \mathrm{eV}$.
\end{abstract}

\section{INTRODUCTION}

The current design of ITER projects for its active phase tungsten (W) as plasma-facing components (PFCs) in the divertor areas, i.e. the areas receiving the highest particle and energy flux densities. An important issue in this respect remains the physical sputtering of W by impurities, especially when extrinsic impurities are seeded to reduce the power loads on the PFCs. A high local $\mathrm{W}$ source in the divertor may cause unduly high $\mathrm{W}$ concentrations in the centre, cooling of the plasma and thus deterioration of the fusion performance. In the present work, we utilize the ITER-like Wall configuration at JET matching the environment for ITER closely to gain insights into relevant aspects of divertor $\mathrm{W}$ erosion and $\mathrm{W}$ transport into the main plasma. The main ingredients in relation to tungsten sputtering are firstly the identity, the concentration, and the energy of the sputtering particles, secondly the fraction of the sputtered particles that really leaves the surface, i.e., is not promptly redeposited, and thirdly, the efficiency with which the particles reach the core plasma, i.e. divertor screening. This paper will will focus on the first two of these aspects.

The approach is to quantify the $\mathrm{W}$ erosion in dependence of a wide range of low-Z impurity species, divertor particle flux densities and plasma temperatures and thus to allow for an extrapolation to ITER as well as fusion devices beyond. In particular, spectroscopy is performed on $\mathrm{W}$ and $\mathrm{W}^{+}$in the ultraviolet and blue spectral region and experimental photon efficiencies from TEXTOR are used to quantify the local $\mathrm{W}$ source strength. A con- sistency analysis is performed by comparing the various spectral lines as well as by placing the source strengths in the context of the spectrally determined impurity species distribution and simple erosion estimates using Bohdansky's formula for low-energy ion sputtering, and similar experiments performed at ASDEX Upgrade with its full$\mathrm{W}$ wall. Experiments in $\mathrm{H}$-mode are used to gain insight in the ratio between inter- and intra-ELM sputtering. Finally, impurity seeding is employed to assess the trade-off between introducing more sputtering particles and lowering the impact energy thereof by plasma cooling.

\section{DIAGNOSTICS AND METHODS}

Tungsten erosion was quantified at the horizontal solid tungsten tile in the JET outer divertor on the basis of passive emission spectroscopy. The general idea is that sputtered $\mathrm{W}$ atoms will interact with the (ionizing) divertor plasma in front of the target and therefore either be excited or ionized. Assuming that no atoms escape from the plasma before being ionized, the particle flux can be inferred from an absolutely measured atomic line intensity using the so-called $S / X B$ method [1]. A detailed description of the method can be found in e.g. [2]. Briefly, the (spatially integrated) intensity of a spectral line $\Phi$ is characterized by an excitation rate $X$ to the upper level of the corresponding transition, the branching ratio $B$ of the transition, and the number of atoms in the plasma. The number of atoms is determined by the ionization rate $S$ and the atom influx $\Gamma$. Here, all rates 
generally depend on plasma temperature. Thus the W atom influx can be written as a function of the line intensity:

$$
\Gamma_{W}=\frac{S}{X B}\left(T_{e}\right) \times \Phi
$$

The $S / X B$ multiplication factor is the so-called inverse photon efficiency or the number of ionizations per photon. We used the following formula to incorporate its dependence on the plasma temperature $T_{e}$ as was found by recent experiments at the TEXTOR tokamak in combination with experimental results from other devices [3]:

$$
\frac{S}{X B}\left(T_{e}\right)=53.7\left(1-1.04 \exp \left(-\frac{T_{e}}{22.1}\right)\right) .
$$

It is noted that generally a constant value of $(S / X B)=$ 20 is used, e.g. by Dux et al. [4].

Several spectroscopic systems were used to monitor the WI line radiation at $400.9 \mathrm{~nm}$ at the horizontal target in the JET outer divertor. Primarily, a re-designed mirrorlink system viewing the target from the top of the vessel and analyzing the light in three wavelength ranges with Czerney-Turner spectrometers was used [5], to which we will refer as KT3. The system was typically set (unless otherwise stated) to provide every $0.04 \mathrm{~s}$ a spectrum covering a wavelength range of 395-409 $\mathrm{nm}$ for 20 lines of sight covering $360 \mathrm{~mm}$ of the outer divertor. In a second system, the outer divertor area is viewed, again from the top of the vessel, in 10 lines of sight, each covering $\sim 20$ $\mathrm{mm}$ diameter spots at the divertor target, and relayed by fiber optics to Photo Multiplier Tubes equipped with $400.9 \mathrm{~nm}$ bandpass filters (1 $\mathrm{nm}$ bandwidth), providing a time response of up to $10 \mathrm{kHz}$ (referred to as PMTs or to one of four tunable Czerney-Turner spectrometers (referred to as KS3 [6]. A schematic of the lines of sight of the three spectroscopic systems is shown in Fig. 1.

The same spectroscopy systems and approach were applied to analyze hydrogen and impurity fluxes to the target. The following lines and $(S / X B)$ values (which were selected to reflect a typical divertor plasma temperature of $20-30 \mathrm{eV}$, as was encountered in the experiments presented here) were used:

$$
\begin{aligned}
\text { H } \varepsilon(397 \mathrm{~nm}): & S / X B=1.4 \times 10^{4} \\
\text { Be II }(527 \mathrm{~nm}): & S / X B=70 \\
\text { C II }(515 \mathrm{~nm}): & S / X B=10 \\
\text { C III }(465 \mathrm{~nm}): & S / X B=1
\end{aligned}
$$

For hydrogen flux calculations, we additionally multiplied the $S / X B$ value by 2 in order to take the effect of molecules into account [7].

Finally, Langmuir probe measurements were used to determine profiles of the divertor plasma temperature as well as the incident plasma flux densities. We performed a consistency check on the integrated outer divertor particle flux (i.e. integrated over the strike point on the horizontal solid tungsten target plate) inferred from probe measurements and from Balmer epsilon intensity profiles.

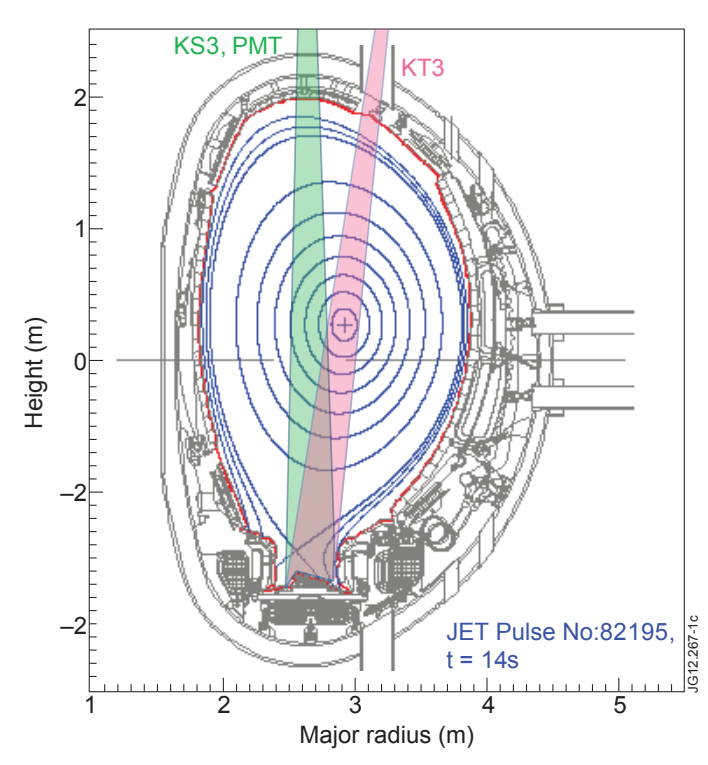

FIG. 1: Cross section of JET in which the lines of sight monitored by the various spectroscopy systems are indicated. The KT3 system covers a chord of $360 \mathrm{~mm}$ long and therewith the horizontal target plate by 20 lines of sights in a mirror linked system. The KS3 and PMT systems view 10 chords of 20 $\mathrm{mm}$ diameter using fiber optics, therewith also covering the horizontal target plate.

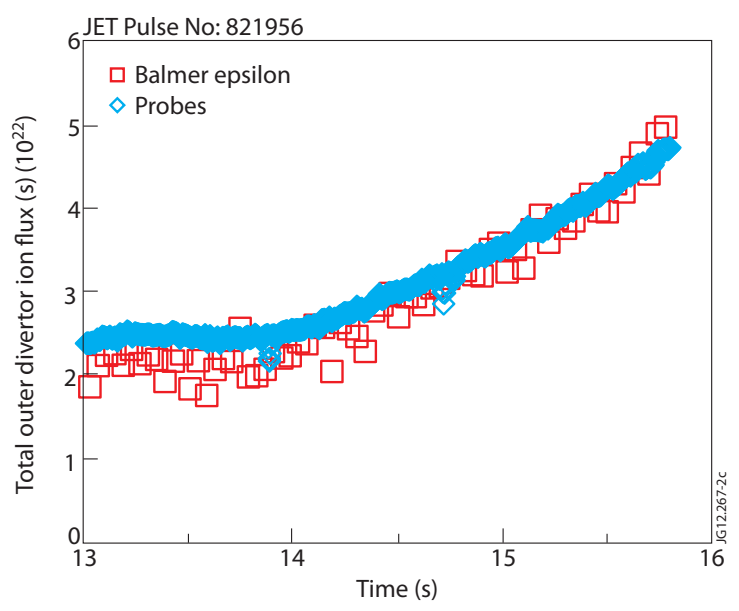

FIG. 2: Comparison of the outer divertor particle flux inferred from Langmuir probe measurements and from Balmer- $\varepsilon$ line intensity measurements.

The result is plotted in Fig. 2 for discharge JPN 82195 and shows that the two approaches agree within $25 \%$.

\section{ASSESSMENT OF THE MAIN SPUTTERING PARTICLE IN L-MODE EXPERIMENTS}

The tungsten erosion in the JET outer divertor was evaluated as a function of the divertor electron tempera- 


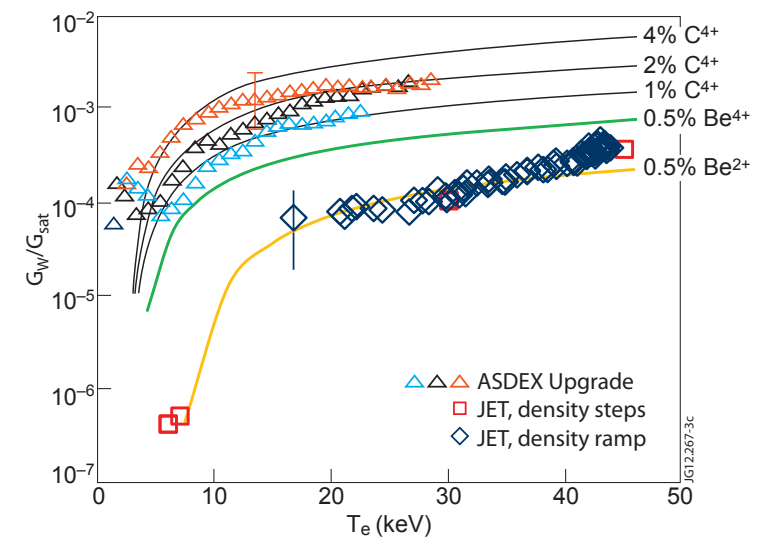

FIG. 3: Effective erosion yields measured in the outer divertor in L-mode with 1 MW NBI heating. The yields are compared with data measured in ASDEX Upgrade for sputtering in $\mathrm{H}-$ mode in-between ELMs from Dux et al.[4] as well as with the erosion yield for different concentrations and charge states of beryllium and carbon.

ture in L-mode discharges with 1 MW auxiliary heating by neutral beams. Data were obtained from three discharges. One was a scan of the divertor temperature from $43 \mathrm{eV}$ down to $17 \mathrm{eV}$ by means of a scan of the divertor fueling rate (JPN 82195). Two discharges contained each three steps in the divertor density in which the divertor temperature was finally lowered to $7 \mathrm{eV}$ (JPN 81474, JPN 81486). The tungsten particle flux densities at the peak of the profile were normalized to the saturation current measured by Langmuir probes at the same radial position. This number shall referred to as the "effective" erosion yield, as is common practice, to express that the majority particle (deuterium) is not the sputtering particle. The results are plotted in Fig. 3 as a function of the divertor plasma temperature that was measured by the same probe. For comparison, the sputter yield is indicated for an admixture of different concentrations of $\mathrm{Be}^{2+}$ (the most abundant charge state for $20<T_{e}<35$ $\mathrm{eV}$ ) and $\mathrm{Be}^{4+}$ (the charge state of beryllium closer to the $\mathrm{x}$-point).

The plot shows that the measured effective erosion yield increases in the temperature range $15<T_{e}<45$ $\mathrm{eV}$ but no threshold behavior is encountered in the data from the density ramp. The pulses with the density steps did achieve the tungsten signal to drop to close to the detection limit. These points are indicative of a threshold behavior that corresponds to $\mathrm{Be}^{4+}$. However, it can not be excluded that line blending of the WI $400.9 \mathrm{~nm}$ line with a presently unknown spectral line is significant at these low intensities. The error bar drawn in the figure for the effective erosion yields in JET reflects the two main uncertainties in the analysis: the value of the photon efficiency $S / X B$ (including the underlying uncertainty in the plasma temperature) and the determination of the plasma flux. Both individual errors are estimated to be $30-50 \%$.
It is seen that a fraction of $0.5 \%$ beryllium in the target flux density is sufficient to explain the observed tungsten sputter signal. This number is in excellent agreement with the intensity of the BeII $527 \mathrm{~nm}$ line as was measured with the PMTs. The signal integrated over the entire horizontal target was typically $1.2 \times 10^{18} \pm 2.4 \times 10^{17}$ photons $\mathrm{m}^{-2} \mathrm{~s}^{-1}$, which translates (with $S / X B=70$ ) into $8.4 \times 10^{19}$ beryllium atoms $\mathrm{m}^{-2} \mathrm{~s}^{-1}$. This value corresponds for the actual variation in the target saturation current to a fraction of $0.35 \%$ up to $0.50 \%$. It is noted that the fraction of beryllium in the outer divertor target flux had apparently been lower than the beryllium fraction in the main plasma for these particular discharges. Assuming beryllium as the only impurity in the main plasma, we estimated its concentration from Z-effective, which ranged from 1.2 to 1.4 for the discharges under evaluation. Thus typical Be concentrations in the main plasma were $1.8 \%-3.6 \%$.

We thus conclude that sputtering by beryllium only explains the entire observed tungsten sputter rate. This obviously doesn't necessarily mean that sputtering by other impurities is not important. For the case under evaluation, we therefore assess the contribution of carbon. PMT measurements are available for the CIII 465 $\mathrm{nm}$ line and give $\mathrm{a} \sim 8$ times larger integrated intensity for the horizontal target compared to the BeII $527 \mathrm{~nm}$ line. Multiplied with the respective photon efficiencies of 70 and 1, this implies a factor of 10 lower carbon flux density compared to the beryllium flux density, i.e. a carbon fraction of $\sim 0.05 \%$, of the total particle flux. Assuming the helium-like charge state of $4+$ for the carbon, such a fraction implies that the contribution of carbon to the tungsten sputtering is roughly a factor of 5 lower than the sputtering by beryllium.

Qualitative information, but with more spatial and spectral resolution, was available from the KS3 system. Comparing intensity profiles showed that the spatial profile of the CIII $465 \mathrm{~nm}$ line radiation was much wider than for the BeII $527 \mathrm{~nm}$ line or e.g. the CII $515 \mathrm{~nm}$, the latter two being more alike. The intensity ratio CII/BeII was 0.6 , i.e. a particle flux ratio $\mathrm{C} / \mathrm{Be}$ of 0.08 . These numbers confirm again that sputtering by carbon impurities is negligible compared to sputtering by beryllium.

The unimportance of carbon in the operation of the JET ILW was observed throughout the 2011/2012 JET campaigns as concentrations were generally in the $0.05 \%$ concentration range [8]. We regard this as a demonstration of the beneficial effect of the ITER-like wall configuration on the impurity species distribution in the JET divertor.

Data from the all tungsten ASDEX Upgrade from Dux et al. [4] are included for comparison. These were determined before any boronization had been performed and were put in the perspective of sputtering by carbon impurities as a placeholder for all low-Z impurity ions present in the divertor target particle flux. It shows that the effective tungsten sputter yield in JET under these specific attached divertor conditions is less than one tenth of the 
values measured in ASDEX Upgrade in-between ELMs at similar plasma temperatures.

\section{EFFECT OF INCREASING THE BERYLLIUM CONTENT IN LOW DENSITY L-MODE DISCHARGES WITH LARGE SAWTEETH}

After having established that beryllium is the dominant impurity with respect to tungsten sputtering, we investigated the dependence of the tungsten sputtering on both the beryllium flux and the divertor plasma conditions in a larger parameter range. For that reason, discharges with 1-3 MW ICRH auxiliary heating at medium to low line averaged plasma density $\left(1.6-2.8 \times 10^{19} \mathrm{~m}^{-3}\right)$ were analyzed. Characteristic of these discharges were the strong oscillations in the divertor plasma temperature (and target flux density), which is illustrated by the time traces plotted in Fig. 4 for discharge JPN 80896. The upper trace is the oscillation in the divertor plasma temperature that was measured with Langmuir probes. The lower trace shows the raw as well as smoothed PMT measurement of the WI radiation at the strike point. The figure illustrates that the WI photon flux follows the temperature oscillations closely. This gives the opportunity to relate the instantaneous WI photon (or particle) fluxes and divertor conditions and evaluate again the effective erosion yields as a function of plasma temperature, i.e. energy of the sputtering particle. The results of this analysis is plotted in Fig. 5 for the discharges JPN80889, 80893 , and 80896. It is seen that the increase of the tungsten sputtering with divertor temperature is similar as before, but also depended on the total heating power and on the main plasma density. The effect of the heating power relates to an increasing Be content in the plasma and thus increasing Be influx in the divertor. Both the analysis of Z-effective of the main plasma and the BeII photon fluxes in the outer divertor indicate this. However, the observed increases in Z-effective did not account for the observed increase in tungsten sputtering at the lower densities. It presently remains unclear what effect is responsible. Possible effects playing a role here include uncertainties in the relation between the Langmuir probes temperature measurements and the impact energy of the sputtering particles, a plasma density dependence of the WI $S / X B$ values, the appearance of other sputtering impurities or changes in the (non-coronal) charge state distributions.

\section{ASSESSING PROMPT REDEPOSITION VIA WII SPECTROSCOPY}

A consequence of the grazing angle incidence of the magnetic field with the divertor target is that most of the sputtered particles will return to the surface when the ionization mean free path of the sputtered tungsten

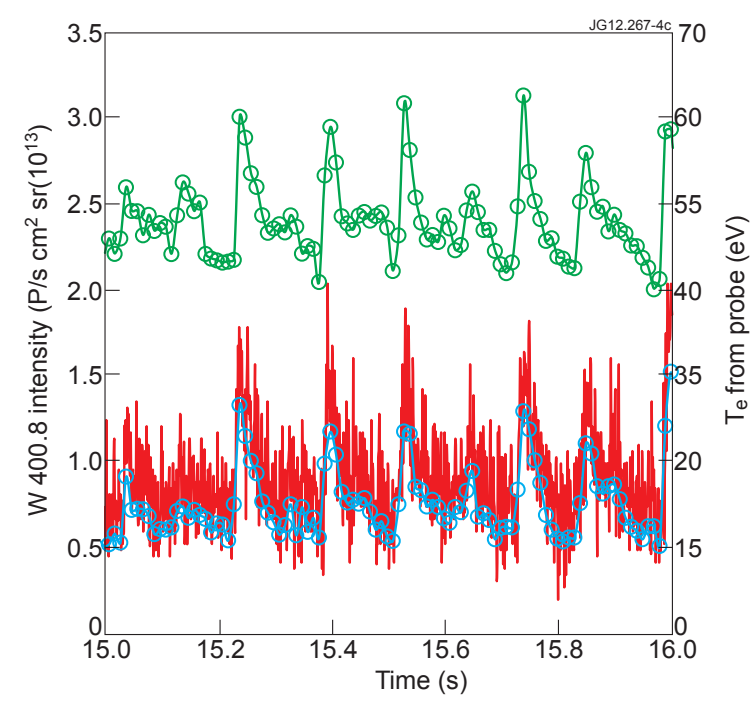

FIG. 4: Time traces of the divertor plasma temperature measured with Langmuir probes (upper signal, plotted against the right horizontal axis) and the WI line radiation measured with a PMT (bottom signal against the left horizontal axis) for discharge JPN 80896. The $20 \mathrm{eV}$ temperature variations are promptly followed by the tungsten photon flux.

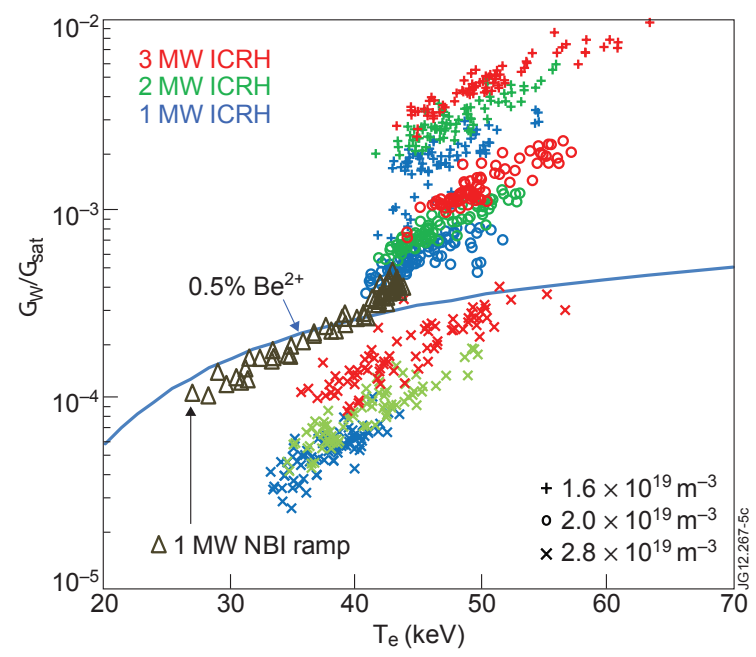

FIG. 5: Effective erosion yields determined from the sawteeth dominated ICRH discharges (JPN80889, 80893, 80896). The symbols refer to different main plasma densities, the colors refer to the different additional heating levels. For comparison, also the data from Fig. 3 are included.

atoms becomes smaller than the ion Larmor radius. This effect has first been discussed by Fussmann et al. and Naujoks et al. [9, 10]. Writing the redeposited fraction as

$$
f_{\text {prompt }}=\left[1+\left(\tau_{\text {ion }} \omega_{c}\right)^{2}\right]^{-1},
$$

and thereby assuming a $\cos \theta$ angular velocity distribution of the sputtered atoms and correctly averaging over the distribution of ionization times for all ionization states 


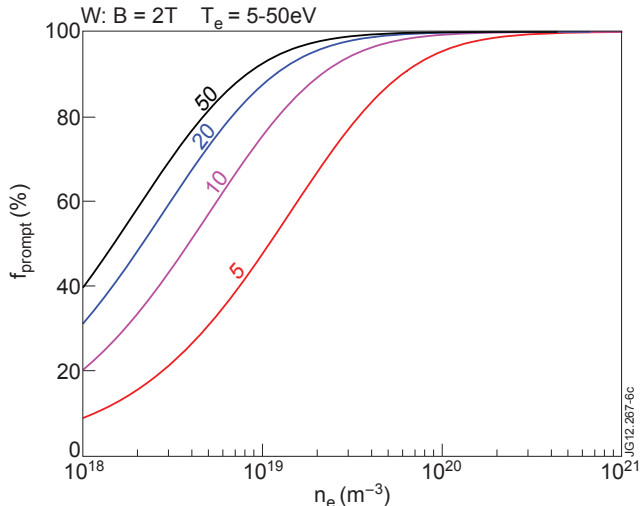

FIG. 6: Redeposited fraction calculated with eqn 4 for conditions similar to the present experiments in JET. Assumed was a $\cos \theta$ angular velocity distribution of the sputtered atoms.

allows to calculate the fraction as a function of divertor temperature and density. We note that this does not take into account contributions from plasma drag [11] or presheath electric fields [12] into account and thus represents a lower limit for the prompt redeposition. For a magnetic field of $2.0 \mathrm{~T}$, which was typical for the JET experiments discussed in the present paper, this resulted in the dependence plotted in Fig. 6. The plot shows that the redeposited fraction changes strongly at divertor densities of around $10^{19} \mathrm{~m}^{-3}$ given the temperature regime typical for the discharges discussed above, i.e. $20<T_{e}<50 \mathrm{eV}$.

A possible way to experimentally characterize the promptly redeposited fraction is by comparing spectral lines of neutral tungsten and ionized tungsten [13]. The rationale is that the $S / X B$ for neutral tungsten lines is unaffected by redeposition "losses", whereas these do form an addition loss channel for ionized particles. Thus, assuming a redeposition rate $R$, the photon efficiency $(S / X B)_{i o n}$ transforms into $((S+R) / X B)_{i o n}$.

Suitable WII lines are found in the UV, e.g. at 364 nm. A spectrum recorded in discharge JPN 80896 around that wavelength is shown in Fig. 7. In addition to the tungsten lines, also chromium and nickel lines are recognized (from Inconel support structures in the main vessel, probably transiently deposited at the strike point position). The two highlighted spectral lines were selected for the WII/WI line ratio analysis. The WI $361.7 \mathrm{~nm}$ line was selected on basis of its high intensity as well as strong correlation with the WI $400.9 \mathrm{~nm}$ line. The WII was selected for its relatively high intensity as well as for available $S / X B$ values from modeling [2]. For the present analysis, we have not applied the calculated S/XB values because the uncertainty in the tungsten ground state temperature introduces an uncertainty in the $\mathrm{S} / \mathrm{XB}$ value of an order of magnitude. Instead, the line intensity ratios $\Phi_{W I I} / \Phi_{W I}$ were evaluated for the discharges JPN 80893 and 80896 around $t=20 \mathrm{~s}$, which means for divertor electron densities of $0.4 \times 10^{19} \mathrm{~m}^{-3}$ and $1.2 \times 10^{19}$ $\mathrm{m}^{-3}$, respectively (measured by Langmuir probes at the

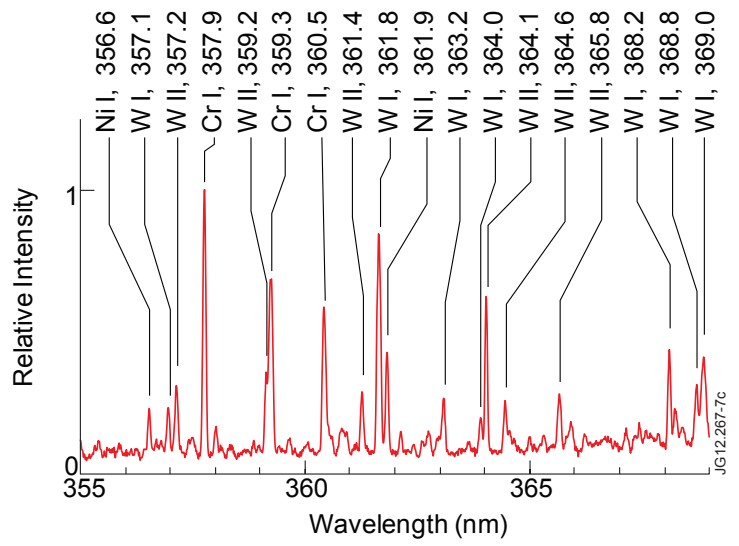

FIG. 7: Identification WI and WII lines in a UV spectrum recorded in discharge JPN 80896.

strike point). The measured divertor temperature was $50 \mathrm{eV}$ and the same for both line ratios (essential for the present evaluation). The line ratio's were $2.1 \pm 0.2$ and $1.1 \pm 0.3$, respectively (note the difference in relative accuracy due to the much lower line intensities at the higher plasma density). In other words, indeed a significant change is observed in the line intensity ratio.

The observed change in the line intensity ratio can be put in the context of a change in the promptly redeposited fraction by assuming that the two density cases differ only in the promptly redeposited fraction and in the tungsten sputter rate. We find using the effective inverse photon efficiency $(S+R) / X B$ for the two density cases $a$, i.e. $\left.0.4 \times 10^{19} \mathrm{~m}^{-3}\right)$, and $b$, i.e. $1.2 \times 10^{19} \mathrm{~m}^{-3}$ :

$$
\frac{\left(\Phi_{W I I} / \Phi_{W I}\right)_{a}}{\left(\Phi_{W I I} / \Phi_{W I}\right)_{b}}=\frac{S+R_{b}}{S+R_{a}}=1.9
$$

With this relation, a minimum value for the redeposited fraction at density $b$ is easily estimated by setting the redeposition rate $R_{a}=0$. It yields that $R_{b}=0.9 S$, i.e. $\sim 50 \%$ of the particles is promptly redeposited. We note the agreement of this value with results from local injection of $W F_{6}$ at the twin limiters in TEXTOR under similar $n_{e}$ and $T_{e}$ conditions, which yielded a promptly redeposited fraction of $50 \%$ [13]. Similarly, it is evaluated how well the observations agree with the calculated fractions for a divertor temperature of $50 \mathrm{eV}$ in Fig. 6, i.e. $78 \%$ and $90 \%$, respectively. These percentages correspond to $R_{a}=3.5 \times S$ and $R_{b}=9 \times S$, respectively, and thus to a ratio $\left(S+R_{b}\right) /\left(S+R_{a}\right)=2.2$, i.e. close to the observations.

\section{TUNGSTEN SPUTTERING IN H-MODE: INTER- VERSUS INTRA-ELM SPUTTERING}

The large difference between inter- and intra-ELM W sputtering in $\mathrm{H}$-mode is illustrated by the tungsten sputter rate profiles that are shown in Fig. 8. Obviously, 


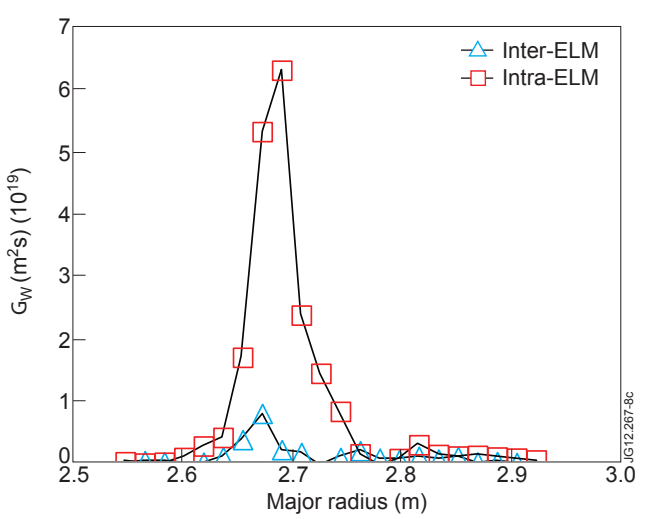

FIG. 8: Comparison of the time inter- and intra-ELM tungsten sputtering shows that intra-ELM sputtering is dominant. The outer divertor tungsten sputter rate profiles were measured in discharge JPN 82237 (13 MW NBI, $7.5 \times 10^{19} \mathrm{~m}^{-3}$ line averaged $n_{e}, 10 \mathrm{~Hz}$ ELMs).

the time resolution at which the spectra underlying these profiles were recorded was insufficient to resolve ELMs given the $40 \mathrm{~ms}$ time resolution of the KT3 system. Therefore, an example was chosen in which the ELM frequency was sufficiently low to record (by coincidence) spectra containing the contribution of one single ELM and spectra without any ELM contribution. The PMT measurements confirmed this for the profiles presented.

For the specific example, the inter-ELM sputtering amounted to $6.3 \times 10^{18}$ atoms/s (following from integration over the entire outer strike point). The interELM total saturation current in the outer divertor was $1.7 \times 10^{23} \mathrm{el} / \mathrm{s}$, which means that the effective inter-ELM sputter yield amounted $4 \times 10^{-5}$ and was thus similar to the L-mode sputter yields that were determined above. The intra-ELM sputtering was $5.7 \times 10^{18}$ atoms/ELM (subtracting the inter-ELM sputtering from the profiles containing the intra-ELM sputtering). Given the $10 \mathrm{~Hz}$ ELM frequency, this means that intra-ELM sputtering dominated by a factor of 9 over inter-ELM sputtering.

\section{NITROGEN SEEDING FOR CONTROLLING TUNGSTEN SPUTTERING}

Impurity seeding to reduce power and energy loads on divertor targets (e.g. [14, 15] and references therein) will add extrinsic impurities to the species mix. It is evident that, within the context of divertor target material sputtering, this involves a balance between increasing the impurity concentration due to the seeding on the one hand and decreasing the divertor plasma temperature to reduce the impact energy of the sputtering particle at the other hand. We illustrate this balance on the basis of a series of pulses (JPN 82293-82296: 1.1 MW NBI heating) in which nitrogen was seeded at different rates $\left(4 \times 10^{21}-1 \times 10^{22} \mathrm{el} / \mathrm{s}\right)$ in the divertor. The nitrogen flux to the target was monitored via the NIII $403.5 \mathrm{~nm}$ line.
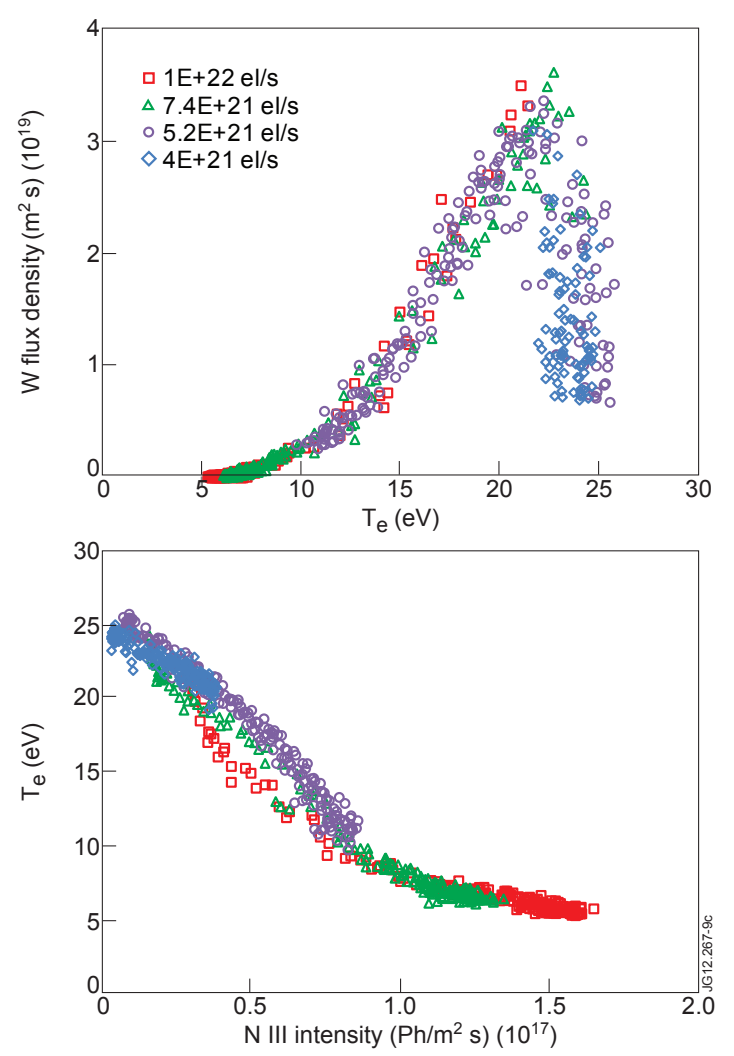

FIG. 9: Effect of nitrogen seeding in the divertor. The lower panel shows how the divertor plasma temperature drops as the nitrogen fluxes build up. The top panel shows that this leads initially to a steep increase in the tungsten sputtering due to higher concentrations impurities (i.e. nitrogen), whereafter plasma cooling takes over to decrease the sputtering.

This signal is observed to build up during the discharge although the seeding level is being kept constant, a feature that is generally known for nitrogen seeding. The result is that the divertor plasma temperature decreases during the discharge. The lower panel in Fig. 9 shows the temperature decrease from initially $25 \mathrm{eV}$ at the lowest seeding rate down to finally $5 \mathrm{eV}$ at the highest seeding rate as a function of the NIII line intensity. The upper panel in the figure shows the tungsten particle flux determined from the WI line intensity as a function of the plasma temperature as it has been set by the nitrogen seeding. The plot confirms the expected trend. Starting from the highest temperatures, the tungsten sputtering increases steeply upon nitrogen seeding, evidently due to sputtering by nitrogen. In the present example the increase is a factor of three. The effect of plasma cooling starts to dominate around $20 \mathrm{eV}$, i.e. where the maximum sputtering is observed. The nitrogen seeding starts to have a net beneficial effect, i.e. a reduction in the tungsten erosion, when the temperature drops below 15 $\mathrm{eV}$. 


\section{CONCLUSIONS}

The tungsten source in the outer divertor of JET in its ITER-like wall configuration, which is the region in which tungsten sputtering is maximum, has been quantified under various experimental conditions from spectroscopic observation of the horizontal target plate that consists of solid tungsten lamellae. We conclude that the erosion is mainly caused by beryllium, which is also the dominant impurity in the plasma. An estimate from spectroscopic signals indicates that the carbon fraction in the target flux is only $0.05 \%$. In the one example studied in $\mathrm{H}$-mode, it was confirmed that sputtering during ELMs dominates over in-between ELM sputtering. A calculation of the promptly redeposited tungsten fraction predicts an order of magnitude lower net erosion, i.e. number of particle that really leave the target surface, compared to the gross erosion that has been characterized in the present work. The redeposited fraction has been assessed by comparing neutral tungsten and tungsten ion lines and confirms that prompt redeposition is significant.

\section{Acknowledgement}

This work, supported by the European Communities under the contract of Association between EURATOM and FOM, was carried out within the framework of the European Fusion Development Agreement. The views and opinions expressed herein do not necessarily reflect those of the European Commission.
[1] K. Behringer et al., Plasma Phys. Control. Fus. 31 (1989) 2059.

[2] A. Pospieszczyk et al., J. Phys. B: At. Mol. Opt. Phys. 43 (2010) 144017.

[3] M. Laengner et al., this volume.

[4] R. Dux et al., J. Nucl. Mater. 390 - 391 (2009) 858.

[5] A. Meigs et al., Rev. Scientific Instrum. 81 (2010) $10 \mathrm{E} 532$.

[6] J. Nucl. Mater. 337-339 (2005) 1038.

[7] Brezinsek et al., Plasma Phys. Control. Fus. 47 (2005) 615.
[8] S. Brezinsek et al, this volume.

[9] G. Fussmann et al., Plasma Physics and Controlled Nuclear Fusion Research, 1995 (Proc. 15th Int. Conf. (Seville 1995)) vol 2 (Vienna: IAEA).

[10] D. Naujoks. et al., Nucl. Fusion 36 (1996) 671.

[11] Stangeby et al., this volume.

[12] D. Tskhakaya et al., this volume.

[13] S. Brezinsek et al., Phys. Scr. T145 (2011) 014016.

[14] J. Rapp et al., Nucl. Fusion 44 (2004) 312-319.

[15] C. Giroud et al., Nucl. Fusion 52 (2012) 063022. 